\title{
Extending Fuzzy Semantic Model by Advanced Decision Rules
}

\author{
Lassaad Ellouze, Rafik Bouaziz \\ MIRACL, Multimedia InfoRmation systems and Advanced \\ Computing Laboratory \\ FSEGS, Route de l'Aérodrome, BP 1088, 3018 Sfax, \\ Tunisia \\ las_ellouze@yahoo.fr; raf.bouaziz@fsegs.rnu.tn
}

\author{
Salem Chakhar \\ LAMSADE, University of Paris Dauphine \\ Place du Maréchal de Lattre de Tassigny \\ 75775 Paris Cedex 16, France \\ salem.chakhar@dauphine.fr
}

\begin{abstract}
This paper extends FSM, a recently proposed semantic data model that supports fuzziness, imprecision and uncertainty of real-world. More precisely, the paper proposes four new concepts, decisional grouping, inhibition, multiplicity and selection, which allows enhancing the modeling of real-world applications. It integrates these concepts in FSM by the definition of new decision rules.
\end{abstract}

\section{Keywords-fuzzy database, decision rule, fuzzy semantic model}

\section{INTRODUCTION}

Different works have been interested to model and manipulate fuzzy, uncertain and imprecise data [10,1,2]. Most of these works introduce fuzziness only at the attribute level and consider that entities are fully encapsulated into their classes. This is very restrictive in many data-intensive applications (e.g. geographical and environmental information systems, decision support systems) in which it is often difficult to assign an entity to a particular class, mainly when this entity verifies only partially the properties of this class. So, several proposals to extend object-oriented and semantic database models have been proposed to support fuzziness, uncertainty and imprecision at the class definition level $[6,5,7,11,9,8,3]$. An entity may then be partially a member of its class according to a given degree of membership (d.o.m). These data models propose different formula to compute the d.o.m. The fuzzy semantic model (FSM) [3] defines the concept of decision rules, which are the basis for computing these degrees. However, FSM, as the other models, fails to support some semantic particularities relative to the computing of the d.o.m, which are very important in several applications.

The objective of this paper is to enrich FSM by some additional concepts to permit a more realistic computing of membership degrees. More precisely, the paper defines four new concepts, decisional grouping, inhibition, multiplicity and selection, which allows improving the modeling of real-world applications. It gives solutions to express these concepts in FSM by the definition of new decision rules.

The paper is organized as follows. Section II presents briefly the FSM and compares it to other proposals. Sections III-VI present the proposed extended concepts, which are successively: decisional grouping, inhibition, multiplicity and selection. Section VII concludes the paper.

\section{RELATED WORK}

\section{A. Fuzzy semantic model}

FSM allows taking into account uncertainty and imprecision both at the attribute and the class levels. It authorizes an entity to be partially member of its class according to a given d.o.m.

A fuzzy class in FSM is a collection of fuzzy entities having some similar properties. Fuzziness is thus induced whenever an entity verifies only (partially) some of these properties. In FSM we design by $X_{K}=\left\{p_{1}, p_{2}, \ldots, p_{n}\right\}(\mathrm{n} \geq 1)$ the set of properties which define a fuzzy class $K$. These properties may be derived from the attributes of the class and/or from common semantics. Each of them defines what is designed by decision rule in FSM formalism. Attribute-based decision rules are of the form: $\langle$ Attribute_Name $\rangle\langle o p\rangle\langle$ value $\rangle$ where $o p$ is a binary or a set operator. Operator $o p$ may be crisp or fuzzy. The degree to which each of these properties determines fuzzy class $K$ is not the same. To ensure this, we associate to each property $p_{i}$ a non-negative weight $w_{i}$ reflecting its importance in deciding whether or not an entity $e$ is a member of a given fuzzy class K. The d.o.m of the fuzzy entity $e$ in fuzzy class $K$ is:

$$
\mu_{K}(e)=\frac{\sum_{i}^{n} \rho_{p_{k}^{i}}\left(v_{i}\right) \cdot \omega_{i}}{\sum_{i}^{n} \omega_{i}},
$$

where $v_{i}$ is the value of the attribute on which property $p_{i}$ is based, for entity $e$, and $\rho_{p_{k}^{i}}\left(v_{i}\right)$ is the partial membership function relative to property $p_{i}$. Equation (1) is a generic formula, used as a basis to compute the membership degrees for different FSM constructs (association, generalization, specialization, aggregation, grouping and composition relationships, subclass/superclass relationships) (see $[3,4]$ for more details).

Figure 1 illustrates an extract from a FSM model relative to the medical domain and concerns the multiple sclerosis "MS" disease; it is a progressive demyelinization disease that progresses by periods of remissions and relapses, and whose cause remains not fully known. This model contains four classes: Patient, Consultation, Diagnostic type and MS. Each patient is characterized by three simple and crisp attributes 
(Birth_Date, Name and Address) and a derived fuzzy attribute (Age). She/He can perform different consultations at different dates. According to the symptoms observed in a given consultation, different diagnostic types may be associated with appropriate appurtenance degrees. The latter are computed according to the level of verification of their proprieties. The fuzzy class $M S$ is composed of attributes Beginning_Age, Tiredness_Symptoms and MS_Type. It has two decision rules based on attributes Beginning_Age and Tiredness_Symptom:

$$
X_{M S}=\{\text { Beginning_Age } \geq \text { young, Tiredness_Symptoms }=h i g h\}
$$

The weights of these decision rules are 0.5 and 0.6 , respectively.

Example. Consider an aged patient $e_{1}$ for which the propriety Beginning Age zyoung is fully verified. The doctor observes that this patient has a recent significant tiredness symptom and values by 0.9 the level of verification of propriety Tiredness_Symptoms $=$ high. This patient is defined as $e_{1}=\left\{\left(1, p_{1}\right),\left(0.9, \bar{p}_{2}\right)\right\}$. The membership degree of entity $e_{1}$ to class MS, for this example, is computed as follows:

$$
\mu_{M S}\left(e_{1}\right)=\frac{1 * 0.5+0.9 * 0.6}{0.5+0.6}=0.945 \text {. }
$$

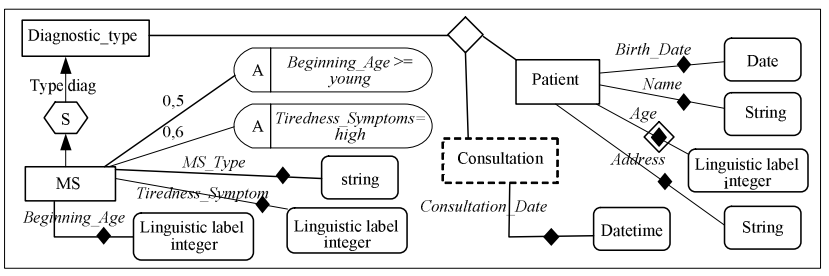

Figure 1. Extract from FSM model relative to multiple sclerosis " $M S$ "

\section{B. Other models}

Since Enhanced FSM must support all concepts of FSM, i.e. it must ensure the bottom-up compatibility, it differs from other models of literature by the same elements. To define the d.o.m of an object in its class, the authors in [11] use a weighted sum of the inclusion degrees of the attribute values in the attribute ranges as they are defined at the class level. They use the relevance of attributes to classes as weights. In the proposal of [8], the authors use a weighted sum of the inclusion degrees of the attribute values in the attribute domains where the importance of attributes to classes are used as weights. The inclusion degrees are computed differently in these two proposals. FSM uses the partial d.o.m instead of the inclusion degrees. The weights in the three proposals have similar interpretations. However, in $[11,8]$ all the attributes of the class are used to compute the d.o.m (although, an attribute can have zero as weight in order to eliminate it from consideration) but FSM can consider only a subset of attributes.

The extended FSM as it will be described in this paper is distinct from all these models by four new concepts. The concept of decisional grouping allows aggregating several decision rules when their joint presence has an important effect on the computing of the d.o.m of entities to classes. The concept of decisional inhibition allows modeling the cases where a decision rule inhibits partially or totally another decision rule. The concept of decisional multiplicity is useful to take into account situations where the presence of a decision rule may be optional or multiple (i.e. it can have 0 or more instances). Finally, the concept of decisional selection allows identifying the decision rule to use for computing the d.o.m.

\section{DeCisional GROUPING CONCEPT}

In the current version of FSM, attribute-based decision rules are based on one attribute only. There is no way to define rules requiring several attributes. Figure 2 shows, through an example, the limitation of FSM decision rules definition. Indeed, among MS symptoms we may cite the Neurological disorders and the resolutive onset. Taken separately, the attributes modeling these two symptoms have low weights $(0.3$ and 0.2 , respectively). But when jointly present, these symptoms enhance the probability that the patient has MS. In this case, a much higher common weight, that may reach 0.9, should be used. But in FSM we can only define two distinct decision rules; each one is associated with one symptom, which is clearly not fully realistic. To avoid this limitation, we propose to add to FSM formalism the concept of decisional grouping, defined below.

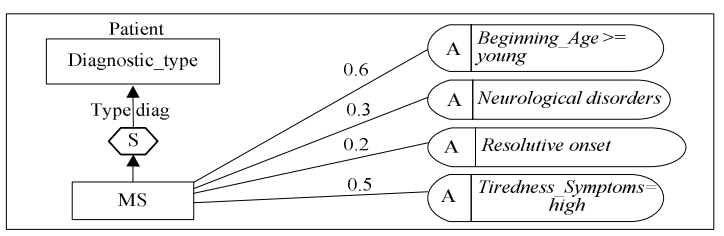

Figure 2. Limitation of FSM decision rules

Definition 1: Decisional Grouping concept. The decisional grouping is a means to put together several decision rules that act as a composed decision rule with a common weight.

Note that a grouping does not inhibit the individual weights associated with the different grouped decision rules. Indeed, these weights still participate to compute the d.o.m of the entity: to the class when there is no joint effect; to the grouping otherwise.

Proposition 1. The decisional grouping concept is graphically represented by a node related to the considered class by an arc and annotated by the common weight. The grouped decision rules are linked to this common node with different arcs, each is annotated by the weight associated with the decision rule.

Proposition 2. In the case of joint presence of different decision rules, to compute the d.o.m of an entity $e$ into a class $K$, we consider each node as decision rule having as weight the common weight and as value the d.o.m of this entity to this node. The computing of the d.o.m of an entity $e$ to a node is similar to the computing of the d.o.m of an entity to a class, since each node can be seen as an intermediate (virtual) class.

To ensure more flexibility to the application of the grouping concept, we propose to associate it, if necessary, with the totality constraint.

Proposition 3. When the number of grouped decision rules is more than two, a totality constraint may be defined on the 
The 28th North American Fuzzy Information Processing Society Annual Conference (NAFIPS2009)

Cincinnati, Ohio, USA - June 14 - 17, 2009

decisional grouping, qualified hence as a total, to require the joint presence of all decision rules. The absence of one of the decision rules in the total grouping inhibits the effect of this grouping. When this constraint is not mentioned, the grouping applies even if some decision rules are absent. The totality constraint is graphically schematized by the letter $\mathrm{T}$ preceding the common weight. total.

Note that the grouping of two decision rules is implicitly

Proposition 4. The set $X_{K}$ should be extended, when necessary, to include the nodes participating the constitution of the class $K: X_{K}=\left\{p_{1}, p_{2}, \ldots, p_{m}, n_{1}, n_{2}, \ldots, n_{r}\right\} ;(\mathrm{m}+\mathrm{r} \geq 1)$. Accordingly, the d.o.m of an entity $e$ into class $K$ is computed as follows:

$\mu_{K}(e)=\frac{\sum_{i, p_{i} \in X_{K}} \rho_{p_{k}^{i}}\left(v_{i}\right) \cdot \omega_{i}+\sum_{i, n_{i} \in X_{K}} \mu_{n_{i}}(e) \cdot \omega_{n_{i}}^{\text {State }}}{\sum_{i=1}^{m} \omega_{i}+\sum_{i=m+1}^{r} \omega_{n_{i}}^{\text {State }}}$

Where $\rho_{p_{k}^{i}}\left(v_{i}\right)$ is the partial d.o.m relative to property $p_{i}$, $\mu_{n_{i}}(e)$ the d.o.m relative to node $n_{i}$ and $\omega_{n_{i}}^{\text {State }}$ is the weight of node $n_{i}$, computed according to the state of grouping $n_{i}$ for entity $e$. The d.o.m of a node $e$ is computed in a similar way to the computing of the d.o.m of an entity to a class, by applying Equation (2). The weight $\omega_{n_{i}}^{\text {State }}$ is equal to: $(i)$ the common weight of the grouping, when the state is defined by a joint presence of the grouped decision rules in the case of total grouping, or by a not total grouping; (ii) the sum of weights of the grouped decision rules, otherwise. This permits to inhibit the effects of the grouping and grouped decision rules are considered as if they were directly related to the class.

$$
\omega_{n_{i}}^{\text {State }}=\left\{\begin{array}{cl}
\omega_{n_{i}} & \text { if case (i) } \\
\sum_{j, p_{j} \in X_{n_{i}}} \omega_{j}+\sum_{j, n_{j} \in X_{n_{i}}} \omega_{n_{j}}^{\text {State }} \quad \text { if case (ii) }
\end{array}\right.
$$

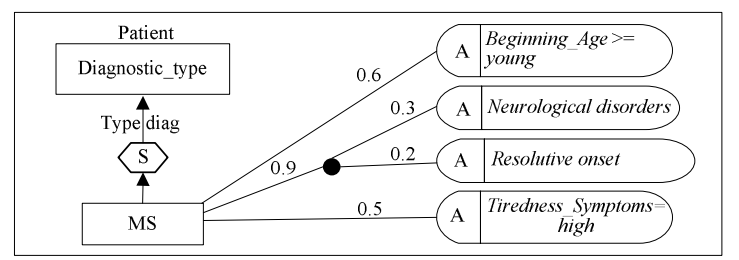

Figure 3. Example of decisional grouping

Consider again the example of Figure 2 where two decision rules relative to the symptoms Neurological disorders and resolutive onset have been defined. The modeling of joint presence of these symptoms through the concept of decisional grouping is given in Figure 3, where a common weight of 0.9 is associated with node $n_{l}$, a weight of 0.3 is associated with the first decision rule and a weight of 0.2 is associated with the second decision rule.

Let $e_{1}$ and $e_{2}$ be two patients defined as follows:

$e_{1}=\left\{\left(0.9, p_{1}\right),\left(0.7, p_{2}\right),\left(1, p_{3}\right),\left(1, p_{4}\right)\right\}$;

$e_{2}=\left\{\left(0.9, p_{1}\right), \quad\left(0, p_{2}\right),\left(1, p_{3}\right),\left(1, p_{4}\right)\right\}$

where $p_{1}, p_{2}, p_{3}$ and $p_{4}$ represent respectively the decision rules associated with the symptoms Neurological disorders, resolutive onset, Tiredness and Beginning_Age. Note that only $e_{1}$ is characterized by a joint presence of symptoms $p_{1}$ and $p_{2}$.

The application of Equation (2) to this example gives:

$\mu_{M s}(e)=\frac{\rho_{p_{M S}^{\prime}}(e \text {.Tiredness_Symtoms }) \cdot \omega_{1}+\rho_{p_{P_{L S}^{2}}}(e . . \text { Beginning_Age }) \cdot \omega_{2}+\mu_{n_{1}}(e) \cdot \omega_{n_{1}}^{\text {State }}}{\omega_{1}+\omega_{2}+\omega_{n_{1}}^{\text {State }}}$

It gives for $e_{1}$ and $e_{2}$ the following d.o.m:

$$
\begin{gathered}
\mu_{M S}\left(e_{1}\right)=\frac{(1 * 0.5)+(1 * 0.6)+\left(\frac{(0.9 * 0.3)+(0.7 * 0.2)}{0.3+0.2} * 0.9\right)}{0.5+0.6+0.9}=0.919 \\
\mu_{M S}\left(e_{2}\right)=\frac{(1 * 0.5)+(1 * 0.6)+\left(\frac{(0.9 * 0.3)+(0 * 0.2)}{0.3+0.2} *(0.3+0.2)\right)}{0.5+0.6+(0.3+0.2)}=0.856
\end{gathered}
$$

\section{CONCEPT OF DECISIONAL INHIBITION}

A decision rule in FSM participates necessarily, with its entire weight, in the computing of d.o.m of entities to classes. However in real-world applications, the effects of certain decision rules and/or decisional groupings may be, partially or fully, inhibited by the presence of other decision rules or groupings. Nevertheless, these specifications can not be expressed under the current version of FSM. Let us come back to the example relative to the joint presence of the two symptoms Neurological disorders and resolutive onset. In reality, the effect of their joint presence reaches the value of 0.9 only in the case of the absence of febrile situation, as high temperature. The presence of high temperature does not only weaken the effect of the grouping, but it also inhibits it considerably in the computing of the d.o.m in respect to class MS.

To model this particularity under FSM, we can only define a new decision rule that favors the absence of febrile situation, with a high weight, 0.95 for instance, as illustrated in Figure 4. This decision rule constitutes so with the initial grouping, that fully participates $\left(\omega_{n_{i}}=1\right)$, a new grouping $n_{2}$. However, this solution does not model the reality faithfully. On the one hand, it reduces insufficiently the d.o.m of the two symptoms at the level of node $n_{2}$ when the febrile situation holds. On the other hand, it takes into account the new decision rule whatever the value of node $n_{l}$; the new rule participates in the computing of the d.o.m of an entity $e$ into class MS even when $\mu_{n_{1}}(e)=0$. In that case, this rule does not ensure a role of inhibition, but intervenes rather as a factor of decision for the whole part.

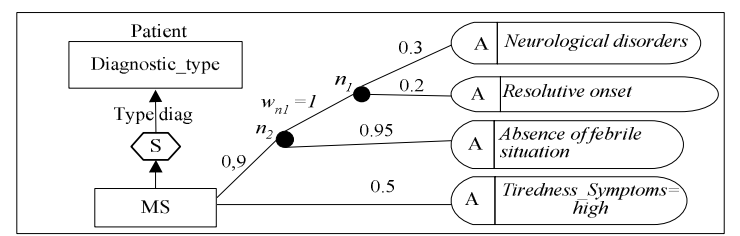

Figure 4. Example showing the need of inhibition

Let $e_{1}, e_{2}, e_{3}$ and $e_{4}$ be four patients defined as follows:

$e_{1}=\left\{\left(0.9, p_{1}\right),\left(0.7, p_{2}\right),\left(0.9, p_{3}\right),\left(1, p_{4}\right)\right\} ; e_{2}=\left\{\left(0.9, p_{1}\right)\right.$, $\left.\left(0.7, p_{2}\right),\left(0.1, p_{3}\right),\left(1, p_{4}\right)\right\} ; e_{3}=\left\{\left(0, p_{1}\right),\left(0, p_{2}\right),\left(0.9, p_{3}\right)\right.$, 
Cincinnati, Ohio, USA - June 14 - 17, 2009

$\left.\left(1, p_{4}\right)\right\} ; e_{4}=\left\{\left(0, p_{1}\right),\left(0, p_{2}\right),\left(0.1, p_{3}\right),\left(1, p_{4}\right)\right\}$, where $p_{1}, p_{2}, p_{3}$ and $p_{4}$ represent respectively the symptoms Neurological disorders, resolutive onset, absence of febrile situation and tiredness. Note that only $e_{1}$ and $e_{3}$ are characterized by the absence of febrile situation.

Patients $e_{1}$ and $e_{2}$ are both characterized by a joint presence of symptoms $p_{1}$ and $p_{2}$ and differ only by $p_{3}$ : absence of symptom febrile situation for $e_{1}$ and presence of this symptom for $e_{2}$. Equation (2) applied to $e_{1}$ and $e_{2}$ gives the following values:

- The partial membership degrees at the level of node $n_{2}$ : $\mu_{n_{2}}\left(e_{1}\right)=0.8597 ; \mu_{n_{2}}\left(e_{2}\right)=0.4666$.

- The partial membership degrees at the level of the class MS: $\mu_{M S}\left(e_{1}\right)=0.9098 ; \mu_{M S}\left(e_{2}\right)=0.6571$.

The d.o.m of $e_{1}$ in respect to node $n_{2}\left(\mu_{n_{2}}\left(e_{1}\right)\right)$ and the d.o.m of $e_{1}$ in respect to MS $\left(\mu_{M S}\left(e_{1}\right)\right)$ are high; these d.o.m remains correct. But the value of $\mu_{n_{2}}\left(e_{2}\right)$ (resp. the value of $\left.\mu_{M S}\left(e_{2}\right)\right)$ shows that the decreasing of the d.o.m of $e_{2}$ to $n_{2}$ (resp. to MS) is not sufficient despite the presence of febrile situation symptom. Concerning patients $e_{3}$ and $e_{4}$, they are characterized by the absence of symptoms $p_{1}$ and $p_{2}$ and equally differ only by the symptom $p_{3}$. Equation (2) gives for these two patients two incorrect partial membership degrees $\left(\mu_{M S}\left(e_{3}\right)=0.553 ; \mu_{M S}\left(e_{4}\right)=0.248\right)$ This shows that the new rule participate wrongly in the computing of the d.o.m despite the absence of $p_{1}$ and $p_{2}$, and even in the presence of febrile situation symptom.

To permit a decision rule to inhibit the effect of another rule while avoiding a direct participation in the computing of the membership degrees, we propose to enrich FSM with the concept of decisional inhibition, hereafter defined.

Definition 2: Decisional inhibition concept. The concept of decisional inhibition is a means to inhibit the effects of a decision rule or a group of decision rules in the computing of the membership degrees. Inhibition may be total or partial and can be fuzzy according to a weight of inhibition. It requires the definition of an inhibition rule associated to the decision rule or to the group of rules to inhibit. The inhibition rule may be composed of a single decision rule or a group of inhibition rules, forming an inhibitor node.

Note that inhibition has sense only if the decision rules to inhibit are verified, i.e. have non null satisfaction degrees.

Proposition 5. The concept of decisional inhibition is graphically represented by a circle including the symbol $\mathrm{I}^{-}$and associated with an arrow from the inhibitor rule towards the inhibited rule or grouping of rules. The circle is labeled by the inhibition weight.

Proposition 6. To evaluate the effect of inhibition on a decision rule or on a node, the new value (after inhibition) is computed as follows:

$$
v^{I^{-}}=v_{\text {afterinhibition }}=v_{\text {to inhibite }} *\left(1-\left(v_{\text {inhibitor }} * w_{\text {inhibition }}\right)\right)
$$

where $v_{\text {inhibitor }}$ represents either the value of the attribute of the inhibitor property in the case of a single inhibition rule, or the partial membership degree $\mu_{n_{i}}(e)$ relative to the inhibitor node $n_{i}$, in the case of a grouping. The multiplication of inhibitor value relative to an entity $e, v_{\text {inhibitor }}$, by the weight of inhibition $w_{\text {inhibition }}$ of the inhibitor rule or grouping of rules, determines the percentage of inhibition for this entity. Finally, the multiplication of the weight to inhibit by the non inhibition percentage (complement of the inhibition percentage: 1 - $\left.\left(v_{\text {inhibitor }} * w_{\text {inhibition }}\right)\right)$ gives the value after inhibition of the final appurtenance degree.

Proposition 7. The d.o.m of an entity $e$ to a class $K$ is computed as follows:

$$
\mu_{K}(e)=\frac{\sum_{i, p_{i} \in X_{K}} \rho_{p_{k}^{i}}^{\left[I^{-}\right]}\left(v_{i}\right) \cdot \omega_{i}+\sum_{i, n_{i} \in X_{K}} \mu_{n_{i}}^{\left[I^{-}\right]}(e) \cdot \omega_{n_{i}}^{\text {State }}}{\sum_{i=1}^{m} \omega_{i}+\sum_{i=m+1}^{r} \omega_{n_{i}}^{\text {State }}}
$$

The notion of "State" is the same as the one defined in Proposition 4 for Equation (2). [I] allows taking into account the presence of inhibition rules. In this case, we may find $\rho_{p_{k}^{i}}^{\left[I^{-}\right]}\left(v_{i}\right)$ and $/$ or $\mu_{n_{i}}^{\left[I^{-}\right]}(e)$, corresponding to the values after inhibition $\quad\left(v_{\text {after inhitition }}\right)$, computed using Equation (3).

Figure 5 uses the concept of decisional inhibition to avoid the limitation of the model in Figure 4. Indeed, the enhanced model permits, in the case of the presence of febrile situation, to inhibit considerably the effect of the grouping of $p_{1}$ and $p_{2}$, Neurological disorders and resolutive onset, in the computing of the d.o.m. In addition, it permits to obtain coherent results when $p_{1}$ and $p_{2}$ are absent.

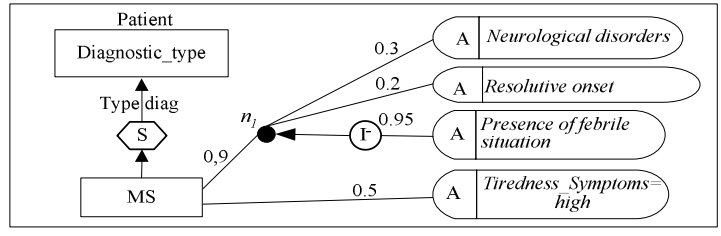

Figure 5. Example of inhibition

In addition, in Figure 5 the values of $p_{3}$ are complementary to those of Figure 4. This is because $p_{3}$ represents here the presence, and not the absence, of the febrile situation symptom. In fact, $p_{3}$ is no longer modeled as decision rule that favors the absence of febrile situation, but as an inhibition rule.

Concerning patients $e_{1}, e_{2}, e_{3}$ and $e_{4}$, having as values $(0.1$, $0.9,0.1,0.9)$ for $p_{3}$, we obtain:

- Partial membership degrees at the level of node $n_{1}$, relative to $p_{1}, p_{2}$ et $p_{3}$, by applying Equations (2) and (3): $\mu_{n_{1}}\left(e_{1}\right)=\mu_{n_{1}}\left(e_{2}\right)=0.82 ; \mu_{n_{1}}\left(e_{3}\right)=\mu_{n_{1}}\left(e_{4}\right)=0 ; \mu_{n_{1}}^{I^{-}}\left(e_{2}\right)=0.119 ;$ $\mu_{n_{1}}^{I^{-}}\left(e_{1}\right)=\mu_{n_{1}}\left(e_{1}\right) *\left(1-\left(v_{p_{3}}\left(e_{1}\right) * w_{p_{3}}\right)\right)=0.82 *(1-(0.1 * 0.95))=0.742$.

- Partial membership degrees in respect to class MS, by applying Equation (4): $\mu_{M S}\left(e_{1}\right)=0.834 ; \mu_{M S}\left(e_{2}\right)=0.4335$; $\mu_{M S}\left(e_{3}\right)=0.3571 ; \mu_{M S}\left(e_{4}\right)=0.3571$.

The values of $\mu_{n_{1}}^{I^{-}}\left(e_{2}\right)$ shows the importance of the inhibition effect on node $n_{1}$ in the case of the presence of febrile situation symptom, which can be equal to zero in the 
case of a total inhibition. $\mu_{M S}\left(e_{2}\right)$ decreases then to about $50 \%$. Moreover the same value of $\mu_{M S}\left(e_{3}\right)$ and $\mu_{M S}\left(e_{4}\right)$ shows that the inhibitor rule does not participate in the computing of the d.o.m of entities $e_{3}$ and $e_{4}$ in respect to class MS when the decision rules to inhibit are not satisfied at all. This shows that the addition of the inhibition concept to FSM allows modeling reality more fruitfully.

Inhibition may also be based on a group of decision rules. Figure 6 gives an example of inhibition group based on decision rules Aged and Unilateral pains, which inhibits the effect of decision rule Attack pains. In fact, in the case of strong presence of this group, it is rather the Trigeminal neuralgia disease that is the most probable.

Let patient $e_{1}$ be defined as $e_{1}=\left\{\left(0.9, p_{1}\right),\left(0.4, p_{2}\right),\left(1, p_{3}\right)\right.$, $\left.\left(0.9, p_{4}\right)\right\}$, where $p_{1}, p_{2}, p_{3}$ and $p_{4}$ represent respectively the decision rules Aged, Unilateral pains, Attack pains and Tiredness. Conformingly to the model of Figure 6 , the decision rule $p_{3}$ is inhibited by the presence of decision rules $\left(p_{1}, p_{2}\right)$, grouped in node $n_{l}$, with an inhibition weight equal to 0.8 .

$$
v_{p_{3}}^{I^{-}}\left(e_{1}\right)=v_{p_{3}}\left(e_{1}\right) *\left(1-\left(\mu_{n_{1}}\left(e_{1}\right) * w_{\text {inibition }} p_{3}\right)\right)=1 *\left(1-\left(\frac{0,9 * 1+0,4 * 0,9}{1,9} * 0,8\right)\right)=0,469 \text {. }
$$

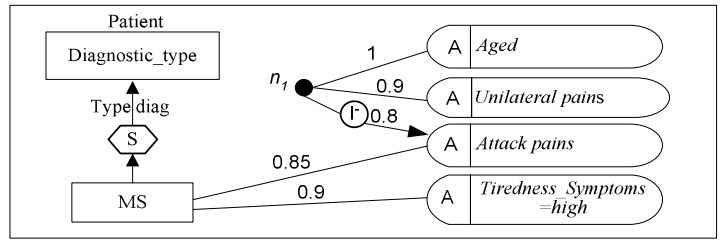

Figure 6. An inhibition based on a group of decision rules

Following the inhibition, the satisfaction level of decision rule Attack pains decrease from 1 to 0.469 conformingly to Equation (3). Based on inhibition, it is possible not only to decrease the degree of satisfaction of the decision rule, but also to totally inhibit its effect. This is the case when the inhibition weight is equal to 1 and the inhibition decision rules are fully satisfied, i.e.: $v_{\text {after inhibition }}=v_{\text {to inhibition }} *(1-(1 * 1))=0$, which is not possible under the initial version of FSM.

\section{CONCEPT OF DeCisional MultiPlicity}

Under the current version of FSM, a decision rule participates exactly one time in the computing of the d.o.m. There is no way to take into account the cases where optional or multiple participation of decision rules even that these situations may hold in real-world applications. Indeed, it is interesting to be able to take advantage of a given decision rule when its satisfaction level is not null, while the absence of this rule does not put into question the computing of appurtenance degrees. Moreover, the multiple presence of certain decision rules can either increase the partial membership degree of entities, or condition the definition of grouped decision rules.

Let us consider the case of optional participation for class MS. The three decision rules Motor system disorder, Sensitivity disorders and MRI shown lesions (both optional and multiple) participate in the computing of the d.o.m, with high weight ( $0.7,0.8$ and 1 , respectively), only if these decision rules have non-null values. However, in the case of the absence of values (i.e. intermediate values and not null values), these decision rules should not participate in the computing of the d.o.m. Nevertheless, it is not possible to model these specifications in the current version of FSM.

To be able to model the optional or multiple participation of decision rules, we propose to add to FSM the concept of decisional multiplicity, defined below.

Definition 3: Concept of decisional multiplicity. This concept is a means to make possible the optional or multiple participation of a decision rule in the computing of the d.o.m with different weights. In the case of multiple participation, an appropriate weight is associated to each instance. In addition, a minimal number of instances may be defined.

Proposition 8. The concept of decisional multiplicity is graphically represented by a set of weights associated with the concerned decision rule $\left(r_{i}\right)$. This set takes the form of $\left\{\omega_{1}^{r_{i}}, \omega_{2}^{r_{i}}, \ldots, \omega_{n}^{r_{i}}\right\}$. If all the weights have the same value, we can simply add the symbol "*" before this value. If the rule is optional, its weight or set of weights is written in square bracket. In addition, if a minimal number of instances is required, this number should be put before the set of weights.

Figure 7 shows how to model the two optional decision rules Motor system disorder and Sensitivity disorders, and the optional and multiple decision rule MRI shown lesions. Each of these decision rules participates only when its satisfaction level is not null. Consider again the case of entity $e_{1}$ with respective values of 0.9 and 0.6 for the decision rules Motor system disorder and Sensitivity disorders, and the absence of the value for decision rule MRI shown lesions. The dominator in this case is limited to the weight of participant decision rules: $0.8+0.7+0.85+0.9=3.25$ and not $0.8+0.7+0.85+0.9+\mathbf{1}=4.25$.

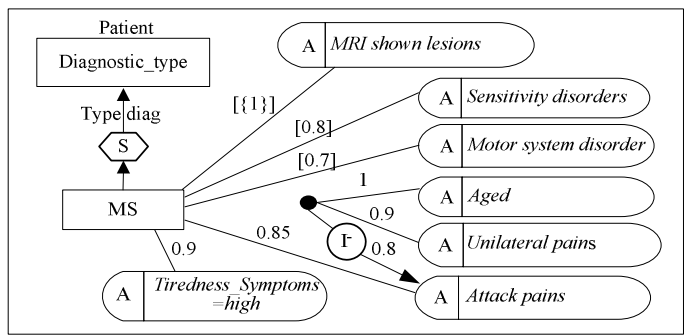

Figure 7. Application of multiplicity concept

The concept of decisional multiplicity may concern a single decision rule or a grouping of decision rules, as shown in Figure 8 , where we can easily distinguish the case of optional decision rule relative to node $n_{4}$ ([1]). This permits to model the case of MS patient with a mono-symptom, which is the MRI shown lesions. In this situation, no other symptom is represented.

Proposition 9. The set $X_{K}$ may contain, when necessary, elements supporting the concept of decisional multiplicity that participate to the definition of class $K$. These elements should be defined in subset $X_{K}^{M}$ The other obligatory and not multiple elements, are to be defined in subset $X_{K}^{O}$. The definition of class $K$ becomes than as follows: $X_{K}=X_{K}^{O} \cup X_{K}^{M}$. Accordingly, the 
The 28th North American Fuzzy Information Processing Society Annual Conference (NAFIPS2009)

Cincinnati, Ohio, USA - June 14 - 17, 2009

d.o.m of an entity $e$ in class $K$ is computed as follows:

$$
\mu_{K}(e)=\frac{\sum_{i, p_{i} \in X_{K}} \psi_{p_{k}^{i}}(e)+\sum_{i, n_{i} \in X_{K}} \psi_{n_{i}}(e)}{\sum_{p_{i} \in X_{K}} \omega_{\psi_{p_{i}}}+\sum_{n_{i} \in X_{K}} \omega_{\psi_{n_{i}}}}
$$

where $\psi_{p_{k}^{i}}(e)$ and $\psi_{n_{i}}(e)$ represent the partial participation functions relative to $p_{i}$ and node $n_{i}$, respectively; and $\omega_{\psi_{p_{i}}}$ and $\omega_{\psi_{n_{i}}}$ represent the weights associated with these functions:

$$
\begin{aligned}
& \psi_{p_{k}^{i}}(e)=\left\{\begin{array}{ll}
\rho_{p_{k}^{i}}^{\left[p^{-}\right]}\left(v_{i}\right) \cdot \omega_{i} & \text { si } p_{i} \in X_{K}^{O} \\
\sum_{j, \exists v_{j}^{i}}^{O} \rho_{p_{k}^{i}}^{\left[I^{-}\right]}\left(v_{j}^{i}\right) \cdot \omega_{j}^{i} & \text { si } p_{i} \in X_{K}^{M} \\
0 & \text { otherwise }
\end{array} \text { and }\left(\begin{array}{l}
\exists \text { Min of instances or } \\
\text { respected Min of instances }
\end{array}\right)\right. \\
& \psi_{n_{i}}(e)=\left\{\begin{array}{ll}
\mu_{n_{i}}^{\left[I^{-}\right]}(e) \cdot \omega_{n_{i}}^{\text {State }}(e) & \text { si } n_{i} \in X_{K}^{O} \\
\sum_{j, \exists \mu_{n_{j}}(e)} \mu_{n_{j}^{i}}^{\left[I^{-}\right]}(e) \cdot \omega_{n_{j}^{i t e}}^{\text {State }} & \text { si } n_{i} \in X_{K}^{M} \text { and } \\
0 & \text { otherwise }
\end{array}\left(\begin{array}{l}
\neg \exists \text { Min of instances or } \\
\text { respected Min of instances }
\end{array}\right)\right.
\end{aligned}
$$

This leads to the following weights that should be used in the participation functions:

$$
\begin{aligned}
& \omega_{\psi_{p i}}= \begin{cases}\omega_{i} & \text { si } p_{i} \in X_{K}^{o} \\
0 & \text { si } p_{i} \in X_{K}^{M} a \text { nd not respected Min of instances } \\
\omega_{1}^{p_{i}} & \text { si } p_{i} \in X_{K}^{M} a \text { nd } p_{i} \text { not optional and } \neg \exists v_{1}^{i} \\
\sum_{j, \exists v_{j}^{j}} \omega_{j}^{i} & \text { otherwise }\end{cases} \\
& \omega_{\psi_{n_{i}}}= \begin{cases}\omega_{n_{i}}^{\text {State }}(e) & \text { si } n_{i} \in X_{K}^{O} \\
0 & \text { si } n_{i} \in X_{K}^{M} a \text { nd not respected Min of instances } \\
\omega_{n_{i}}^{\text {State }} & \text { si } n_{i} \in X_{K}^{M} a \text { nd } n_{i} \text { not optional and } \neg \exists \mu_{n_{1}^{1}} \\
\sum_{j, \exists \mu_{n_{j}^{\prime}}(e)} \omega_{n_{j}}^{\text {State }} & \text { otherwise }\end{cases}
\end{aligned}
$$

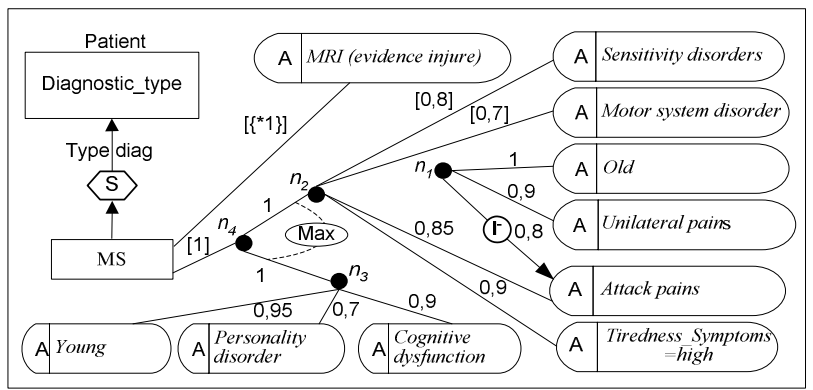

Figure 8. Notion of multiplicity

\section{CONCEPT OF DECISIONAL SELECTION}

In addition to the concept of decisional multiplicity, the model in Figure 8 also presents an application of a new concept: decisional selection. This concept makes possible the selection of the grouping $n_{2}$ or the grouping $n_{3}$, according to a selection criterion, which is the Max operator in this case. Based on this criterion, the grouping having the maximal value is selected. The degree of partial participation of an entity $e$ to node $n_{4}$ is then maximum between the two appurtenance degrees relative of nodes $n_{2}$ and $n_{3}$.
Definition 4. Concept of decisional selection. This concept is a means to select, according to a given criterion (eg. Max or Min operators), a decision rule or a grouping of decision rules among several ones.

Proposition 10. The concept of decisional selection is graphically represented by a discontinued arc comporting a selection criterion. This arc links the set of concerned decision rules and/or grouping of decision rules.

\section{CONCLUSION}

The fuzzy semantic model FSM described in [3] fails to take into account some aspects of real-world applications. In fact, FSM does not permit to handle the following specifications: (i) decision rules that depend on several attributes; (ii) decision rules that may be inhibited by the other decision rules, (iii) optional and multiple participation of decision rules, and (iv) selective application of decision rules (or grouping of decision rules). This paper proposed new concepts (namely, decisional grouping, inhibition, multiplicity and selection) permitting to handle the above cited limitations. Different illustrative examples showed the pertinence of these new concepts.

The proposed solutions permit to better model the realworld aspects. However, additional investigations are required to deal with time-varying constraints. In fact, we intend to further enrich FSM by the temporal dimension; both for data and schema.

\section{REFERENCES}

[1] G. Bordogna, and G. Pasi (Eds.), Recent Issues on Fuzzy Databases. Heidelberg: Physica-Verlag, 2000.

[2] P. Bosc, D. Kraft, and F. Petry, "Fuzzy sets in database and information systems: status and opportunities," Fuz. Sets and Sys., vol 156, pp. 418 426, 2005 .

[3] R. Bouaziz, S. Chakhar, V. Mousseau, S. Ram, and A. Telmoudi, "Database design and querying within the fuzzy semantic model," Inf. Sci., vol 177, no 21, pp. 4598-4620, November 2007.

[4] R. Bouaziz, S. Chakhar, and I. Saad, "Membership functions definition in the fuzzy semantic model," Inter. Conf.: Sci. of Electronic, Technologies of Inf. and Telecommunications (SETIT'05), Mars 27-31, Sousse, Tunisia, 2005.

[5] G.Q. Chen, and E.E. Kerre, "Extending ER/EER concepts towards fuzzy conceptual data modeling," in: Proc. of The IEEE Inter.Conf. on Fuz. Sys., vol 2, Anchorage, Alaska, USA, May 4-9, pp. 1320-1325, 1998.

[6] D. Rocacher, and P. Bosc, "The set of fuzzy rational numbers and flexible querying," Fuz. Sets and Sys., vol 155, pp. 317-339, 2005.

[7] N.V. Gyseghem, and R.D. Caluwe, "Imprecision and uncertainty in UFO database model," J. of American Society of Inf. Sci., vol 49, no 3, pp. 236-252, 1998.

[8] Z.M. Ma, W.J. Zhang, and W.Y. Ma, "Extending object-oriented databases for fuzzy information modeling," Inf. Sys., vol 29 , pp. 421435, 2004.

[9] A. Yazici, B.P. Buckles, and F.E. Petry, "Handling complex and uncertain information in the ExIFO and $\mathrm{NF}^{2}$ data models," IEEE Transa. on Fuz. Sys., vol 7, no 6, pp. 659-676, 1999.

[10] A. Yazici, and R. George, Fuzzy database modeling. Heidelberg: Springer-Verlag, 1999.

[11] A. Yazici, R. George, and D. Aksoy, "Design and implementation issues in fuzzy object-oriented data model," Inf. Sci., vol 108, no 1/4, pp. $241-$ $260,1998$. 